\title{
On $k$-rainbow domination in regular graphs
}

\author{
BOŠTJAN KUZMAN \\ University of Ljubljana, Faculty of Education, \\ and Institute of Mathematics, Physics and Mechanics (IMFM)
}

\begin{abstract}
The $k$-rainbow domination problem is studied for regular graphs. We prove that the $k$-rainbow domination number $\gamma_{r k}(G)$ of a $d$-regular graph for $d \leq k \leq 2 d$ is bounded below by $\lceil k n / 2 d\rceil$, where $n$ is the order of a graph. We determine necessary conditions for regular graphs to attain this bound and find several examples. As an application, we determine exact $k$-rainbow domination numbers for all cubic Cayley graphs over abelian groups.
\end{abstract}

Keywords: Domination; Rainbow domination number; Regular graphs; Cayley graphs. 2000 Mathematics Subject Classification: 05C69, 05C85.

\section{Introduction}

The concept of $k$-rainbow domination, as introduced by Brešar et al. in [2], is an extension of the classical domination problem in graphs and was initiated by Hartnell and Rall [7, who studied the domination numbers of cartesian product $G \square K_{2}$ in relation to the Vizing conjecture. The problem has natural applications in analysis of networks.

Let $G=(V, E)$ be a finite, simple graph, and let $k$ be a given nonnegative integer. We denote by $\mathcal{C}=\{1, \ldots, k\}$ the set of colors. We say that a coloring function $f: V \rightarrow 2^{\mathcal{C}}$ that assigns to each vertex $v \in V$ a subset of colors $f(v) \subseteq \mathcal{C}$, is a $k$-rainbow dominating function (or $k$-RDF) on $G$, if

$$
\forall v \in V: f(v)=\emptyset \Longrightarrow \bigcup_{u \sim v} f(u)=\mathcal{C}
$$

In other words, every non-colored vertex $v \in V$ is $k$-rainbow dominated by its neighbours of all possible colors. For a given $k$-RDF, we define the weight of $f$ as

$$
w(f)=\sum_{v \in V}|f(v)|
$$

The $k$-rainbow domination number $\gamma_{r k}(G)$ is the minimal possible weight attained by a $k$-RDF on $G$ :

$$
\gamma_{r k}(G)=\min \left\{w(f) \mid f: V \rightarrow 2^{\mathcal{C}} \text { is a } k-\mathrm{RDF}\right\}
$$

Any $k$-rainbow domination function $f$ of weight $w(f)=\gamma_{r k}(G)$ is called a $\gamma_{r k}(G)$-function.

Clearly, any 1-rainbow domination function on $G$ corresponds to a usual dominating set for $G$, and 1-rainbow domination number $\gamma_{r 1}(G)$ coincides with the usual domination number $\gamma(G)$ of the graph. Also, it was shown in [2, that $\gamma_{r k}(G)=\gamma\left(G \square K_{k}\right)$, where $G \square K_{k}$ denotes the cartesian product of $G$ with a complete graph on $k$ vertices. For given graph $G$ and positive integer $k$, determination of the exact value $\gamma_{r k}(G)$ is known to be NP-complete [3, 4].

For $k=2,3$, the exact values and upper bounds for $k$-rainbow domination numbers of specific graph families such as the generalized Petersen graphs, trees, products of paths and cycles, grid graphs, etc, were studied in several papers, see for instance [3, 4, 8, 9, 11, 12. Moreover, general upper bounds for $k$-rainbow domination numbers of connected graphs are also known and were proven to be tight for some specific graphs, see [5, 6].

\footnotetext{
0 This work is supported in part by the Slovenian Research Agency (ARRS), research program P1-0285 and research projects J1-9108, J1-1694, J1-1695.

Corresponding author e-mail: bostjan.kuzman@pef.uni-lj.si (B. Kuzman)
} 
However, there are few results available on determining the full set of $k$-rainbow dominaton numbers of particular graph families for all relevant $k$. In order to determine these for some graph $G$, one could first determine $\gamma_{r 1}(G)$ and then apply the following theorem to obtain some lower and upper bounds for $\gamma_{r k}(G)$ for $k \geq 2$.

Theorem 1.1 (Shao et al., 2014). Let $G=(V, E)$ be a connected graph of order $n$.

(i) If $k^{\prime}>k$, then

$$
\gamma_{r k^{\prime}}(G) \leq \gamma_{r k}(G)+\left(k^{\prime}-k\right)\left\lfloor\frac{\gamma_{r k}(G)}{k}\right\rfloor .
$$

(ii) If $\Delta$ denotes the maximal degree in graph $G$, then

$$
\gamma_{r k}(G) \geq\left\lceil\frac{k n}{\Delta+k}\right\rceil .
$$

In this paper, we focus on $k$-rainbow domination numbers of regular graphs. In Section 2 , we prove the following Theorem, which improves the lower bound of Theorem 1.1 for $d$-regular graphs, whenever $d \leq k \leq 2 d$.

Theorem 1.2. Let $G$ be a d-regular graph of order $n$. Then

$$
\gamma_{r k}(G) \geq\left\lceil\frac{k n}{2 d}\right\rceil \text { for } k \leq 2 d
$$

and $\gamma_{r k}(G)=n$ for $k \geq 2 d$.

In Section 2, we also prove several other inequalities and interesting results for $k$-rainbow domination functions of regular graphs. In Section 3, we define a d-rainbow domination regular graph as a $d$-regular graph such that $\gamma_{r k}(G)$ attains the lower bounds from Theorem 1.2 for all $d \leq k \leq 2 d$ and investigate necessary conditions for parameters of such graphs. In Section 4 . we investigate exact $k$-rainbow domination numbers of all connected cubic Cayley graphs over abelian groups for all $k$ and determine all 3-rainbow domination regular graphs among these. In Section 5, some further examples of 4-rainbow domination regular graphs are given and some open questions are posed.

\section{Lower bounds for regular graphs}

Unless otherwise noted, throughout this section, graph $G=(V, E)$ will be regular of order $n$ and degree $d$. By using elementary counting arguments, we shall obtain certain bounds on the weight of any $k$-rainbow domination function $f$ on $G$.

Suppose that graph $G=(V, E)$ and some function $f: V \rightarrow 2^{\mathcal{C}}$ are given. In what follows, we denote the non-disjoint sets of vertices that are colored with color $i \in \mathcal{C}$ by

$$
V_{i}=\{v \in V: i \in f(v)\} \text { for } i=1, \ldots, k,
$$

and by $V_{0}=\{v \in V: f(v)=\emptyset\}$ the set of non-colored vertices. Further, we denote the disjoint sets of vertices which are colored with exactly $i$ different colors by

$$
C_{i}=\{v \in V:|f(v)|=i\} \text { for } i=0, \ldots, k,
$$

their union of all colored vertices by

$$
C=\bigcup_{i=1}^{k} C_{i},
$$

and the sets of edges with exactly $i$ end-vertices colored by

$$
\begin{aligned}
& E_{0}=\{\{u, v\} \in E: f(u)=f(v)=\emptyset\}, \\
& E_{1}=\{\{u, v\} \in E: f(u)=\emptyset \neq f(v)\}, \\
& E_{2}=\{\{u, v\} \in E: f(u) \neq \emptyset, f(v) \neq \emptyset\} .
\end{aligned}
$$

Also, we denote the sizes of respective sets by

$$
e_{i}=\left|E_{i}\right|, \quad n_{i}=\left|V_{i}\right|, \quad c_{i}=\left|C_{i}\right|, \quad c=|C|=n-c_{0} .
$$

Our first observation is obtained by double counting the elements of $E_{1}$ in two different ways, and then omitting some terms to obtain upper and lower bounds for the number of $f$-colored vertices $c$. 
Lemma 2.1. Let $G$ be a d-regular graph of order $n$ and let $f$ be some $k$-rainbow domination function on $G$. Then

$$
e_{1}=c d-2 e_{2}=c_{0} d-2 e_{0}
$$

and the following inequalities hold:

$$
\begin{gathered}
c_{0}-\frac{2 e_{0}}{d} \leq c \leq c_{0}+\frac{2 e_{2}}{d}, \\
\frac{n}{2}-\frac{e_{0}}{d} \leq c \leq \frac{n}{2}+\frac{e_{2}}{d} .
\end{gathered}
$$

Next, we use another double counting argument to get several lower bounds for the weight of a $k$-RDF.

Lemma 2.2. Let $G$ be a d-regular graph of order $n$ and let $f$ be some $k$-rainbow domination function on G. Then the following inequalities hold:

$$
\begin{aligned}
& w(f) \geq \frac{(n-c) k+2 e_{2}}{d}, \\
& w(f) \geq \frac{k n+2 e_{2}}{k+d} \\
& w(f) \geq \frac{(k-d)}{d} n+\frac{2 d-k}{d} c, \\
& w(f) \geq n-\left(\frac{2 d-k}{d}\right) c_{0} .
\end{aligned}
$$

Proof. First, we count the number of ordered triples $(u, v, i)$, where $u \sim v, f(u)=\emptyset$ and $i \in f(v)$. Since each empty vertex is dominated by at least $k$ neighbours of different colors, we see this number is at least $(n-c) k$. On the other hand, there are exactly $w(f)$ pairs $(v, i)$ with $i \in f(v)$, and each of these has $d$ neighbours, some colored, others non-colored. Subtracting twice the number of edges with both edges colored we get the exact number of triples. Therefore, $(n-c) k \leq w(f) d-2 e_{2}$, and inequality (5) follows. Since $w(f) \geq c$, inequality (6) is easily obtained from (5). Eliminating $2 e_{2}$ from inequalities (5) and (3), we combine them to obtain (7) and then rewrite with $c=n-c_{0}$ to obtain (8).

We merge Lemmas 2.1 and 2.2 to obtain the following Proposition.

Proposition 2.3. Let $G$ be a d-regular graph of order $n$ and let $f$ be some $k$-rainbow domination function on $G$. Then

$$
w(f) \geq\left\lceil\frac{k n}{2 d}\right\rceil \text { for } k<2 d,
$$

and $w(f) \geq n$ for $k \geq 2 d$.

Proof. For $k<2 d$, we check two cases. If $c \geq n / 2$, we apply inequality 77 of Lemma 2.2 to get

$$
w(f) \geq \frac{(k-d)}{d} n+\frac{2 d-k}{d} \cdot \frac{n}{2}=\frac{k n}{2 d}
$$

If $c<n / 2$, then $n-c>n / 2$ and we apply Lemma 2.1 to get

$$
w(f)>\frac{k n / 2+2 e_{2}}{d} \geq \frac{k n}{2 d} .
$$

In both cases, $w(f)$ is an integer, greater or equal to $\frac{k n}{2 d}$.

For $k=2 d$, we get $w(f) \geq n$ directly from 77 . For $k>2 d$, we get $w(f) \geq 2(n-c)+\frac{2 e_{2}}{d}$ from (5) and hence $w(f) \geq n$ from $(3)$.

Since for any $\gamma_{r k}(G)$-function $f$ we have $w(f)=\gamma_{r k}(G) \leq n$, Theorem 1.2 is a direct corollary of Proposition 2.3 and needs no further proof. 
Example 2.4. Exact $k$-rainbow domination numbers of cycles $C_{n}, n \geq 3$, were determined for $k=2$ by Brešar and Kraner [3] and for $k=3$ by Shao et al. [9] as

$$
\begin{aligned}
& \gamma_{r 2}\left(C_{n}\right)=\left\lceil\frac{n}{2}\right\rceil+ \begin{cases}1, & n \equiv 2 \quad(\bmod 4), \\
0, & \text { otherwise, }\end{cases} \\
& \gamma_{r 3}\left(C_{n}\right)=\left\lceil\frac{3 n}{4}\right\rceil
\end{aligned}
$$

Using Theorem 1.2, we get $\gamma_{r k}\left(C_{f} n\right)=n$ for all $k \geq 4$ and lower bounds

$$
\gamma_{r 2}\left(C_{n}\right) \geq\left\lceil\frac{n}{2}\right\rceil, \gamma_{r 3}\left(C_{n}\right) \geq\left\lceil\frac{3 n}{4}\right\rceil
$$

which can be used to shorten the original proofs significantly.

We can also rewrite inequality (7) to obtain bounds for parameters $c$ and $c_{0}$.

Proposition 2.5. Let $G$ be a d-regular graph and let $f$ be $a \gamma_{r k}(G)$-function, where $0<k<2 d$. Then

$$
\begin{aligned}
c & \leq \frac{d \gamma_{r k}(G)+(d-k) n}{2 d-k}, \text { and } \\
c_{0} & \geq \frac{d\left(n-\gamma_{r k}(G)\right)}{2 d-k} .
\end{aligned}
$$

\section{$3 \quad d$-rainbow domination regular graphs}

The next theorem gives necessary conditions for a $d$-regular graph to attain the lower bound for $\gamma_{r k}(G)$.

Theorem 3.1. Let $G$ be a d-regular graph of order $n$ and let $k<2 d$. If $\gamma_{r k}(G)=\frac{k n}{2 d}$, then $k \geq d, 2 d \mid n$ and $G$ is bipartite.

Proof. For $k<2 d$, let $w(f)=\frac{k n}{2 d}$ for some $\gamma_{r k}(G)$-function $f$. Then $c<n / 2$ is not possible by 5 and $c \geq n / 2$ forces $c=n / 2$ by (7). Since $\gamma_{r k}(G) \geq c$, we also get $k \geq d$. Moreover, it follows from (5) and (2) that $e_{0}=e_{2}=0$ and $e_{1}=e$, so $G$ is bipartite with bipartition sets $C_{0}, C$ of size $n / 2$. Now, count pairs $(i, u)$, such that $i \in \mathcal{C}, u \in V, f(u)=\emptyset$. Their number equals $v_{i} d$ and is at least $c_{0}=n / 2$, so $v_{i} \geq n / 2 d$. But $w(f)=\sum i v_{i} \geq k n / 2 d$ implies equality $v_{i}=n / 2 d$ for all $i>0$, so $2 d \mid n$. (Observe that the proof also implies that for any $\gamma_{r k}(G)$-function $f$, equality $v_{i}=n / 2 d$ must hold for all colors $i \in \mathcal{C}$.)

Example 3.2. The Franklin graph F in Figure 1 shows that conditions of Theorem 3.1 are not sufficient. Since assigning a color $i \in \mathcal{C}$ to any two vertices in one bipartition set cannot dominate all 6 vertices in the other bipartition set, we get $\gamma_{r 3}(F)>6$.

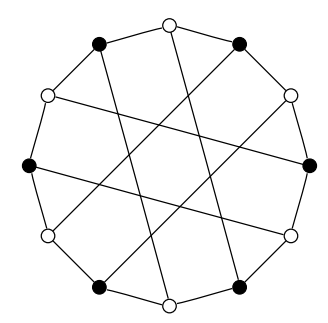

Figure 1: Franklin graph is 3-regular and bipartite with $\gamma_{r 3}(F)>3 n / 2 d$.

Example 3.3. On the other hand, it is easy to see that the complete bipartite graph $G=K_{d, d}$ satisfies conditions of Theorem 3.1 and the equality $\gamma_{r k}(G)=\frac{k n}{2 d}$ holds for all $d \leq k \leq 2 d$. Indeed, for $d \leq k \leq 2 d$, denote by $u_{i}, v_{i} \in V(G)$ the vertices in the bipartition sets and let

$$
f\left(u_{i}\right)=\{j \in \mathcal{C}: j \equiv i \quad(\bmod d)\} \text { and } f\left(v_{i}\right)=\emptyset \text { for } i=1, \ldots, d
$$

Then each color appears exactly once and dominates $d$ non-colored vertices, so $f$ is a $k$-RDF of weight $w(f)=k=k \frac{n}{2 d}$. 
The 'rainbow property' of graphs $K_{d, d}$ described above can be generalized as follows. We define that graph $G$ is d-rainbow domination regular (or $d$-RDR), if it is $d$-regular and equality $\gamma_{r d}(G)=\frac{|V(G)|}{2}$ holds. We now show this also implies a stronger condition.

Proposition 3.4. Suppose that $G$ is a d-regular graph of order $n$, and let $d \leq k<2 d$ for some $k$. If $\gamma_{r k}(G)=\frac{k n}{2 d}$, then $\gamma_{r(k+1)}(G)=\frac{(k+1) n}{2 d}$.

Proof. Suppose that $d \leq k<2 d$ and $f: V \rightarrow\{1, \ldots, k\}$ is $\gamma_{r k}(G)$-function with $\gamma_{r k}(G)=\frac{k n}{2 d}$. Then $v_{i}=\frac{n}{2 d}$ for all $i=1, \ldots, k$ by proof of Theorem 3.1 and a $\gamma_{r(k+1)}$-function $f^{\prime}$ can be explicitly constructed from $f$ by selecting (any) color $i \in\{1, \ldots, k\}$ and adding color $k+1$ to all $i$-colored vertices, that is, defining

$$
f^{\prime}(v)= \begin{cases}f(v) \cup\{k+1\} ; & i \in f(v), \\ f(v) ; & i \notin f(v) .\end{cases}
$$

Obviously, $f^{\prime}$ is a $(k+1)$-RDF of weight $w\left(f^{\prime}\right)=\sum_{i} v_{i}=\frac{(k+1) n}{2 d}$.

Corollary 3.5. Let $G$ be a d-regular graph of order $n$. Then $G$ is d-rainbow domination regular if and only if $\gamma_{r k}(G)=\frac{k n}{2 d}$ for all $d \leq k \leq 2 d$.

Example 3.6. For any $d \geq 1$, complete bipartite graph $K_{d, d}$ is a $d$-RDR graph. It is not difficult to see that a cycle $C_{n}$ is a $2-R D R$ graph if and only if $n \equiv 0(\bmod 4)$.

We shall identify some more examples of $d$-RDR graphs in the next section.

\section{Cubic Cayley graphs over abelian groups}

In this section, we study the $k$-rainbow domination numbers for connected cubic (that is, 3-regular) Cayley graphs over some finite abelian group $H$. Our motivation comes from the fact that Cayley graphs are a large class of regular graphs with nice symmetry properties that could provide some more examples of $d$-RDR graphs, and also from some existing results on values and bounds for 2- and 3-rainbow domination numbers of generalised Petersen graphs that partially overlap with our class and could be extended to larger $k$. For cubic graphs, we readily have $\gamma_{r k}(G) \geq \frac{k n}{6}$ for $3 \geq k<6$ and $\gamma_{r k}(G)=n$ for $k \geq 6$ by Theorem 1.2

Recall that for any finite group $H$ and inverse closed subset $S=S^{-1} \subseteq H \backslash\left\{1_{H}\right\}$, the (non-directed and simple) Cayley graph $G=\operatorname{Cay}(H, S)$ is defined by vertex set $V(G)=H$ and edge set $E(G)=$ $\left\{\{g, h\}: g, h \in H, g h^{-1} \in S\right\}$. Graph $G=\operatorname{Cay}(H, S)$ is regular of degree $d=|S|$ and $G$ is connected if and only if $S$ is a generating set for group $H$.

Note that in order for $\operatorname{Cay}(H, S)$ to be cubic, we must have either $S=\left\{a, a^{-1}, b\right\}$, where $a \neq a^{-1}$ and $b=b^{-1}$, or $S=\{a, b, c\}$, where $a=a^{-1}, b=b^{-1}$ and $c=c^{-1}$. This implies the following well-known classification of such graphs. We omit details of the proof.

Proposition 4.1. Let $G=\operatorname{Cay}(H, S)$ be a connected cubic Cayley graph over some finite abelian group $H$. Then $G$ is isomorphic either to a m-sided prism $\operatorname{Pr}(m)$ or to a Möbius ladder $\operatorname{ML}(m)$, where $m=$ $\frac{|H|}{2} \geq 2$ and graphs $\operatorname{Pr}(m)$ and $\operatorname{ML}(m)$ of order $n=2 m$ both have vertex set $V=\left\{u_{i}, v_{i}: i \in \mathbb{Z}_{m}\right\}$, while the edge set is equal to $E=\left\{\left\{u_{i}, v_{i}\right\},\left\{u_{i}, u_{i+1}\right\},\left\{v_{i}, v_{i+1}\right\}: i \in \mathbb{Z}_{m}\right\}$ for prisms $\operatorname{Pr}(m)$ and to $E=\left\{\left\{u_{i}, v_{i}\right\},\left\{u_{i}, u_{i+1}\right\},\left\{v_{i}, v_{i+1}\right\}: i \in \mathbb{Z}_{m}, i \neq m-1\right\} \cup\left\{\left\{u_{m-1}, v_{m-1}\right\},\left\{u_{m-1}, v_{0}\right\},\left\{v_{m-1}, u_{0}\right\}\right\}$ for Möbius ladders $\operatorname{ML}(m)$.

This reduces the problem to determination of $\gamma_{r k}(G)$ for prisms and Möbius ladders. We shall state and prove separate theorems for each case. Note that the prisms $\operatorname{Pr}(m)$ are just generalised Petersen graphs $G P(m, 1)$, so for $k=2,3$, their $k$-rainbow domination numbers were already determined in [9, 10].

Theorem 4.2. Let $G=\operatorname{Pr}(m)$ be the $m$-sided prism of order $n=2 m, m \geq 3$. Then

$$
\begin{aligned}
& \text { - } \gamma_{r 1}(G)=\left\lceil\frac{m}{2}\right\rceil+ \begin{cases}1 ; & m \equiv 2(\bmod 4), \\
0 ; & m \equiv 0,1,3(\bmod 4) .\end{cases} \\
& \text { - } \gamma_{r 2}(G)=m .
\end{aligned}
$$


- $\gamma_{r 3}(G)=m+ \begin{cases}0 ; & m \equiv 0(\bmod 6), \\ 1, & m \equiv 1,2,3,5(\bmod 6), \\ 2 ; & m \equiv 4(\bmod 6) .\end{cases}$

- $\gamma_{r 4}(G)=\left\lceil\frac{4 m}{3}\right\rceil+ \begin{cases}0 ; & m \equiv 0,1(\bmod 6) \\ 1 ; & m \equiv 2,3,4,5(\bmod 6)\end{cases}$

- $\gamma_{r 5}(G)=\left\lceil\frac{5 m}{3}\right\rceil+ \begin{cases}0 ; & m \equiv 0,1,2,5(\bmod 6) \\ 1 ; & m \equiv 3,4(\bmod 6)\end{cases}$

- $\gamma_{r k}(G)=2 m$ for $k \geq 6$.

Moreover, for $m \equiv 0(\bmod 6)$ and $3 \leq k \leq 6$, the lower bound $\gamma_{r k}(G)=\frac{k m}{3}$ is attained. For $k=4,5$, appropriate $\gamma_{r k}(G)$-functions are given in Table 1 .

Before providing the proof, we state the following observation, which is valid for any graph $G$ (also non-regular) and is sometimes essential for finding exact values of $\gamma_{r k}(G)$.

Lemma 4.3 (Discharging principle for $k$-rainbow domination). If a $\gamma_{r k}(G)$-function $f$ on $G$ has minimal number of non-colored vertices (that is, size $|\{v: f(v)=\emptyset\}|$ is minimal among all $\gamma_{r k}(G)$-functions on $G)$, then

$$
|f(v)| \leq|\{u: u \sim v, f(u)=\emptyset\}|
$$

for all $v \in V(G)$.

Proof. If the condition is not true for some $v \in V$, reassigning ("discharging") some colors from $v$ to its non-colored neighbours reduces $c_{0}$ without changing $w(f)$.

Proof of Theorem 4.2. For $k=1$, the result is well-known [1] and for $k=2,3$, the results are proven in [9, 10. The case $k \geq 6$ is also clear.

Let $k=4$. Using Theorem 1.2 for the lower bound and Theorem 1.1 with $t=3=t^{\prime}+1$ for the upper bound, we obtain

$$
\left\lceil\frac{4 m}{3}\right\rceil \leq \gamma_{r 4}(G) \leq\left\lceil\frac{4 m}{3}\right\rceil+ \begin{cases}0 ; & m \equiv 0,1 \quad(\bmod 6), \\ 1 ; & m \equiv 2,3,5 \quad(\bmod 6), \\ 2 ; & m \equiv 4 \quad(\bmod 6) .\end{cases}
$$

For $m=0,1(\bmod 6)$, we obviously have $\gamma_{r 4}(G)=\left\lceil\frac{4 n}{3}\right\rceil$. For $m=3(\bmod 6)$, the prism $G=\operatorname{Pr}(m)$ is not bipartite, so we have a strict inequality $\left\lceil\frac{4 m}{3}\right\rceil=\frac{4 m}{3}<\gamma_{r 4}(G)$ by Theorem 3.1 , and $\gamma_{r 4}(G)=\left\lceil\frac{4 n}{3}\right\rceil+1$ follows. It is not difficult to find appropriate $\gamma_{r 4}(G)$-functions in these 3 cases, see Table 1 .

For $m \equiv 2,5(\bmod 6)$, suppose that $\gamma_{r 4}(G)=\left\lceil\frac{4 n}{3}\right\rceil$, and let $f$ be some $\gamma_{r 4}(G)$-function. Using (8) we obtain $c_{0} \geq m-1 / 2$, hence $c_{0} \geq m$. Since $m=6 t+2$ or $6 t+5$ and every color on a vertex dominates at most 3 empty vertices, this implies that $n_{i} \geq 2 t+1$ or $n_{i} \geq 2 t+2$, respectively, for $i=1,2,3,4$. But then $w(f) \geq 8 t+4$ or $8 t+8$, which is larger than $\gamma_{r 4}(G)$, a contradiction. Hence $\gamma_{r 4}(G)=\left\lceil\frac{4 n}{3}\right\rceil+1$ and appropriate $\gamma_{r 4}(G)$-functions are easily constructed from the known $\gamma_{r 3}(G)$-functions, see Table 1 and Remark 4.4

Finally, for $m \equiv 4(\bmod 6)$, we have $\gamma_{r k}(G)=\left\lceil\frac{4 m}{3}\right\rceil+a$ with $a=0,1$ or 2 . Suppose first that $a=0$ and let $f$ be some $\gamma_{r k}(G)$-function. Then by (8), we get $c_{0} \geq m-1$. If $c_{0} \geq m=6 t+4$, we have $n_{i} \geq 2 t+2$ for all $i$, so $w(f) \geq 8 t+8>8 t+6=\lceil 4 m / 3\rceil$. So $a=0$ implies $c_{0}=m-1$ and $c=m+1$. From (5) we further obtain $e_{2}=3$ and $e_{0}=0$, so every edge has at least one colored vertex. This implies we may wlog (without loss of generality) suppose that the set of noncolored vertices is $C_{0}=\left\{u_{0}, u_{2}, \ldots, u_{m-2}, v_{1}, v_{3}, \ldots, v_{m-3}\right\}$ (note that $f\left(u_{m-1}\right), f\left(v_{m-1}\right) \notin C_{0}$ ). As in the proof of Theorem 4 in [9], we now consider subsets $\mathcal{P}_{i}=\left\{v_{i}, v_{i+1}, v_{i-1}, u_{i}, u_{i+1}, u_{i-1}\right\}$ for $i \in \mathbb{Z}_{m}$, and denote by $\gamma_{i}=\sum_{v \in \mathcal{P}_{i}}|f(v)|$ the $f$-weight of $\mathcal{P}_{i}$. Observe that $\gamma_{0} \geq 5$, since $u_{0}$ is dominated by 4 colors and $v_{m-1} \neq \emptyset$. Similarily, $\gamma_{m-2} \geq 5$, while $\gamma_{i} \geq 4$ for all other $i$. Since each vertex belongs to exactly three blocks $\mathcal{P}_{i}$, we see that

$$
4 m+2=3 w(f)=\sum_{i} \gamma_{i} \geq 4(m-2)+5 \cdot 2 .
$$

Hence, equality holds and we have $\gamma_{i}=4$ for $i \neq 0, m-2$. In particular, $\gamma_{m-1}=4$ implies $\left|f\left(u_{m-1}\right)\right| \leq 1$ and $\left|f\left(v_{0}\right)\right| \leq 1$, and $\gamma_{0}=5$ further implies $\left|f\left(u_{1}\right)\right|=2$. Continuing, we see that $\left|f\left(v_{4}\right)\right|=\left|f\left(u_{7}\right)\right|=\ldots=$ $\left|f\left(u_{m-3}\right)\right|=2$, while $|f(v)|=1$ for all other vertices from $C$. But now, it is easy to see that any choice of 


\begin{tabular}{|c|c|c|c|c|}
\hline$s$ & $\gamma_{r 4}(G)$ & $f\left(\begin{array}{llllllll}u_{0} & u_{1} & u_{2} & u_{3} & u_{4} & u_{5} & \cdots & u_{m-1} \\
v_{0} & v_{1} & v_{2} & v_{3} & v_{4} & v_{5} & \cdots & v_{m-1}\end{array}\right)$ & $\gamma_{r 5}(G)$ & $f\left(\begin{array}{llllllll}u_{0} & u_{1} & u_{2} & u_{3} & u_{4} & u_{5} & \cdots & u_{m-1} \\
v_{0} & v_{1} & v_{2} & v_{3} & v_{4} & v_{5} & \cdots & v_{m-1}\end{array}\right)$ \\
\hline 0 & $\frac{4}{3} m$ & $\begin{array}{lcccccc}\emptyset & 34 & \emptyset & 1 & \emptyset & 2 & \ldots \\
1 & \emptyset & 2 & \emptyset & 34 & \emptyset & \ldots \\
\end{array}$ & $\frac{5}{3} m$ & $\begin{array}{lccccccc}\emptyset & 345 & \emptyset & 1 & \emptyset & 2 & \ldots \\
1 & \emptyset & 2 & \emptyset & 345 & \emptyset & \ldots \\
\end{array}$ \\
\hline 1 & $\frac{4}{3} m+\frac{2}{3}$ & $\begin{array}{cccccccc}\emptyset & 34 & \emptyset & 1 & \emptyset & 2 & \ldots & 2 \\
1 & \emptyset & 2 & \emptyset & 34 & \emptyset & \ldots & 1 \\
\end{array}$ & $\frac{5}{3} m+\frac{1}{3}$ & $\begin{array}{lccccccc}\emptyset & 345 & \emptyset & 1 & \emptyset & 2 & \ldots & 2 \\
1 & \emptyset & 2 & \emptyset & 345 & \emptyset & \ldots & 1 \\
\end{array}$ \\
\hline 2 & $\frac{4}{3} m+\frac{4}{3}$ & $\begin{array}{ccccccccc}\emptyset & 1 & \emptyset & 34 & \emptyset & 2 & \ldots & \emptyset & 2 \\
34 & \emptyset & 2 & \emptyset & 1 & \emptyset & \ldots & 134 & \emptyset \\
\end{array}$ & $\frac{5}{3} m+\frac{2}{3}$ & $\begin{array}{lccccccccc}\emptyset & 345 & \emptyset & 1 & \emptyset & 2 & \ldots & 3 & 2 \\
1 & \emptyset & 2 & \emptyset & 345 & \emptyset & \ldots & 1 & 4 \\
\end{array}$ \\
\hline 3 & $\frac{4}{3} m+1$ & $\begin{array}{cccccccccc}\emptyset & 34 & \emptyset & 2 & \emptyset & 1 & \ldots & \emptyset & 34 & 1 \\
2 & \emptyset & 1 & \emptyset & 34 & \emptyset & \ldots & 2 & \emptyset & 1 \\
\end{array}$ & $\frac{5}{3} m+1$ & $\begin{array}{cccccccccc}\emptyset & 345 & \emptyset & 1 & \emptyset & 2 & \ldots & \emptyset & 345 & 2 \\
1 & \emptyset & 2 & \emptyset & 345 & \emptyset & \ldots & 2 & \emptyset & 2 \\
\end{array}$ \\
\hline 4 & $\frac{4}{3} m+\frac{5}{3}$ & $\begin{array}{ccccccccccc}\emptyset & 2 & \emptyset & 1 & \emptyset & 34 & \cdots & \emptyset & 12 & \emptyset & 34 \\
1 & \emptyset & 34 & \emptyset & 2 & \emptyset & \cdots & 1 & 3 & 2 & \emptyset \\
\end{array}$ & $\frac{5}{3} m+\frac{4}{3}$ & $\begin{array}{llccccccccc}\emptyset & 2 & \emptyset & 1 & \emptyset & 345 & \cdots & \emptyset & 12 & \emptyset & 345 \\
1 & \emptyset & 345 & \emptyset & 2 & \emptyset & \cdots & 1 & 3 & 2 & \emptyset \\
\end{array}$ \\
\hline 5 & $\frac{4}{3} m+\frac{4}{3}$ & $\begin{array}{cccccccccccc}\emptyset & 34 & \emptyset & 1 & \emptyset & 2 & \cdots & \emptyset & 34 & \emptyset & 1 & 2 \\
1 & \emptyset & 2 & \emptyset & 34 & \emptyset & \cdots & 1 & \emptyset & 2 & \emptyset & 34 \\
\end{array}$ & $\frac{5}{3} m+\frac{2}{3}$ & $\begin{array}{cccccccccccc}\emptyset & 2 & \emptyset & 1 & \emptyset & 345 & \cdots & \emptyset & 2 & 1 & \emptyset & 345 \\
1 & \emptyset & 345 & \emptyset & 2 & \emptyset & \cdots & 1 & 3 & 4 & 2 & \emptyset \\
\end{array}$ \\
\hline
\end{tabular}

Table 1: $\gamma_{r k}(G)$-functions of $G=\operatorname{Pr}(m)$ for $k=4,5$, where $m=6 t+s \geq 3$.

colors forces $f\left(u_{m-1}\right)=f\left(v_{m-2}\right)$, hence $u_{m-2}$ is not dominated by all colors. This contradiction shows $a \geq 1$, but in fact, $a=1$, as we can construct an appropriate 4 -RD function of weight $\left\lceil\frac{4 m}{3}\right\rceil+1$, see Table 1

Now, let $k=5$. Using the known bounds and values for $\gamma_{r 4}(G)$, with some computation we obtain

$$
\left\lceil\frac{5 m}{3}\right\rceil \leq \gamma_{r 5}(G) \leq\left\lceil\frac{5 m}{3}\right\rceil+ \begin{cases}0 ; & m \equiv 0,1 \quad(\bmod 6) \\ 1 ; & m \equiv 2,3,4,5 \quad(\bmod 6)\end{cases}
$$

As in case $k=4$, the values of $\gamma_{r k}(G)$ are obtained trivially for $m \equiv 0,1,3(\bmod 6)$.

For $m \equiv 2,4,5(\bmod 6)$, write $m=6 t+2,4,5$, resp., and suppose that $\gamma_{r k}(G)=\lceil 5 n / 3\rceil=10 t+4,7,9$, respectively. Let $f$ be a $\gamma_{r k}(G)$-function. It follows from (8) that $c_{0} \geq m-a$, with $a=2,1,2$ for $m \equiv 2,4,5$ $(\bmod 6)$, respectively. If $c_{0} \geq m$, we get $n_{i} \geq 2 t+1,2,2$, resp., hence $w(f) \geq 5 n_{i}=10 t+5,10,10$, a contradiction. Similarly, we get that $c=m-1$ is not possible for $m=2,5(\bmod 6)$, while for $m=4$ $(\bmod 6)$ we apply $(5)$ to get $e_{2} \leq 3$. Moreover, it now follows from $(2)$ that $e_{2}-e_{0}=3$, implying that $e_{2}=3$ and $e_{0}=0$. As in case $k=4$, we now wlog suppose that $C_{0}=\left\{u_{0}, u_{2}, \ldots, u_{m-2}, v_{1}, v_{3}, \ldots, v_{m-1}\right\}$ and observe sets $\mathcal{P}_{i}$ with weights $\gamma_{i}$ to get $\gamma_{0}, \gamma_{m-2} \geq 6$ and $\gamma_{i} \geq 4$ otherwise. From $3 w(f)=\sum_{i} \gamma_{i}$ we get that the equalities hold, implying that $\left|f\left(u_{1}\right)\right|=\left|f\left(v_{4}\right)\right|=\ldots=\left|f\left(u_{m-3}\right)\right|=3$ and $|f(v)|=1$ for other $v \notin C_{0}$, implying further that $f\left(v_{0}\right)=f\left(u_{m-1}\right)$, a contradiction. Finally, suppose $c=m-2$ with $m=6 t+2$ or 5 . Repeating above arguments, we get that this case is possible with $e_{2}=6, e_{0}=0$. Inspecting possible sets $\mathcal{P}_{i}$ and weights $\gamma_{i}$, it is now not difficult to construct appropriate $\gamma_{r k}(G)$-functions for both cases.

Remark 4.4. It follows from the proof of Theorem 1 in [9], that whenever equality $\gamma_{r(k+1)}(G)=\gamma_{r k}(G)+$ $\left\lfloor\gamma_{r k}(G) / k\right\rfloor$ holds and a $\gamma_{r k}(G)$-function is known, a $\gamma_{r(k+1)}$-function $f^{\prime}$ can be explicitly constructed from $f$ by selecting color $i \in \mathcal{C}=\{1, \ldots, k\}$ such that $n_{i}=\min \left\{n_{1}, \ldots, n_{k}\right\}$ and adding color $k+1$ to all $i$ colored vertices, that is, defining

$$
f^{\prime}(v)= \begin{cases}f(v) \cup\{k+1\} ; & i \in f(v), \\ f(v) ; & i \notin f(v) .\end{cases}
$$

For Möbius ladders, we obtain very similar results.

Theorem 4.5. Let $G=\operatorname{ML}(m)$ be the Möbius ladder of order $n=2 m, m \geq 2$. Then

- $\gamma_{r 1}(G)=\left\lceil\frac{m}{2}\right\rceil+ \begin{cases}1 ; & m \equiv 0(\bmod 4), \\ 0 ; & m \equiv 1,2,3(\bmod 4) .\end{cases}$

- $\gamma_{r 2}(G)=m$.

- $\gamma_{r 3}(G)=m+ \begin{cases}0 ; & m \equiv 3(\bmod 6), \\ 1, & m \equiv 0,2,4,5(\bmod 6), \\ 2 ; & m \equiv 1(\bmod 6) .\end{cases}$

- $\gamma_{r 4}(G)=\left\lceil\frac{4 m}{3}\right\rceil+ \begin{cases}0 ; & m \equiv 3,4(\bmod 6), \\ 1 ; & m \equiv 0,1,2,5(\bmod 6),\end{cases}$ 
- $\gamma_{r 5}(G)=\left\lceil\frac{5 m}{3}\right\rceil+ \begin{cases}0 ; & m \equiv 2,3,4,5(\bmod 6), \\ 1 ; & m \equiv 0,1(\bmod 6)\end{cases}$

- $\gamma_{r k}(G)=2 m$ for $k \geq 6$.

Moreover, for $m \equiv 3(\bmod 6)$ and $3 \leq k \leq 6$, the lower bound $\gamma_{r k}(G)=\frac{k m}{3}$ is attained. For $k=3,4,5$, appropriate $\gamma_{r k}(G)$-functions are given in Table 3 .

Proof. For $k=1$, the result is well known, see [1. For $k=2$, we can define a 2-RDF $f$ of weight $w(f)=m$ on $G$ by setting $f\left(u_{2 i}\right)=\{1\}, f\left(v_{2 i+1}\right)=\{2\}$ and $f(v)=\emptyset$ otherwise, so $\gamma_{r 2}(G) \leq m$. Now suppose $\gamma_{r 2}(G) \leq m-1$ and let $f$ be an appropriate $\gamma_{r 2}(G)$-function of weight $w(f) \leq m-1$. Note that we can wlog suppose that $f$ has the minimal number of non-colored vertices and apply Lemma 4.3 (Discharging principle) whenever needed. Denote by $\mathcal{P}_{i}=\left\{u_{i}, v_{i}, u_{i+1}, v_{i+1}, u_{i-1}, v_{i-1}\right\}$ and $\gamma_{i}=\sum_{v \in \mathcal{P}_{i}}|f(v)|$ for $i \in \mathbb{Z}_{m}$. Observe first that $\gamma_{i} \geq 0,1$ is not possible, so $\gamma_{i} \geq 2$. Now denote by $\alpha$ the number of blocks $\mathcal{P}_{i}$ with $\gamma_{i}=2$ and by $\beta$ the number of blocks with $\gamma_{i} \geq 4$. Then we have

$$
3(m-1) \geq 3 w(f)=\sum_{i} \gamma_{i} \geq 2 \alpha+4 \beta+3(m-\alpha-\beta),
$$

hence $\alpha \geq 3+\beta$. Now let $\gamma_{i}=2$ and inspect the possible values of $f$ on $\mathcal{P}_{i}$.

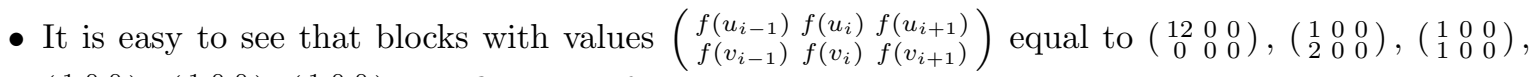
$\left(\begin{array}{lll}1 & 0 & 0 \\ 0 & 0 & 2\end{array}\right),\left(\begin{array}{lll}1 & 0 & 0 \\ 0 & 0 & 1\end{array}\right),\left(\begin{array}{lll}1 & 0 & 0 \\ 0 & 1 & 0\end{array}\right)$ or reflections of such blocks are not possible.

- The blocks of types $\left(\begin{array}{lll}0 & 12 & 0 \\ 0 & 0 & 0\end{array}\right),\left(\begin{array}{lll}0 & 1 & 0 \\ 0 & 2 & 0\end{array}\right),\left(\begin{array}{lll}0 & 1 & 0 \\ 0 & 1 & 0\end{array}\right)$, are possible, but for each of them we have $\gamma_{i \pm 1} \geq 4$, so each their occurence also increases $\beta$. Since $\alpha \geq \beta+3$, we may wlog assume that there are no such blocks.

- Thus, we have at least $\alpha$ blocks of type $\left(\begin{array}{lll}0 & 1 & 0 \\ 2 & 0 & 0\end{array}\right)$ or $\left(\begin{array}{lll}0 & 1 & 0 \\ 0 & 0 & 2\end{array}\right)$. However, for each such block $\mathcal{P}_{i}$ either $\gamma_{i+1}$ or $\gamma_{i-1}$ is at least 4. If $\gamma_{i}=\gamma_{i+1}=2$, then $\gamma_{i-1}=4$ and $\gamma_{i+2}=4$, hence the average weight of these four blocks is 3 . If $\gamma_{i}=2$ and $\gamma_{i+1}=3$, then we must have $\gamma_{i-1}=4$ and $\gamma_{i-2}=3$, and their average weight is again 3. Hence, removing any such quadruplet of blocks from the equation still forces $\alpha \geq \beta+3$, but now there are no more possibilities for $\mathcal{P}_{i}$ with $\gamma_{i}=2$, a contradiction.

Let $k=3$. Then $\lceil n k / 2 d\rceil=m$ is the lower bound for $\gamma_{r 3}(G)$ for all $m$. However, for the lower bound to be exact we must have $6 \mid 2 m$ and $G$ bipartite, which only happens for $m=3(\bmod 6)$. For $m=0,1,4,5(\bmod 6)$, we have $\gamma_{r 3}(G) \geq m+1$, but in fact equality holds, as appropriate functions are easily constructed, see Table 4 .

It remains to show that $\gamma_{r 3}=m+2$ for $m=6 t+1$. Again, by Table 4 we confirm that this is the upper bound. Now suppose $w(f)=m+1=6 t+2$ for some 3 -RDF $f$. Then $c \leq w(f)$ and hence $c_{0} \geq 6 t$. Since each colored vertex dominates at most 3 non-colored vertices, we must have $n_{1}, n_{2}, n_{3} \geq 2 t$, but also $n_{1}+n_{2}+n_{3}=6 t+2$, so $n_{i}=2 t$ for at least one $i$. Wlog assume $n_{3}=2 t$. Then $c_{0} \leq 6 t$ and hence $c_{0}=6 t$ and $c=6 t+2=w(f)$, which implies $c_{1}=c$ and $c_{2}=c_{3}=0$, so each vertex is colored with at most 1 color. By checking all possible cases for adjacent pairs of vertices, that is, $u_{i} \sim v_{i}, u_{i} \sim u_{i+1}$, or $u_{m-1} \sim v_{0}$, it is now easy to see that $f(u)=f(v)=\emptyset$ for some pair $u \sim v$ implies $|f(w)|=2$ for some $w$ adjacent to $u$ or $v$, a contradiction. Thus, we have $e_{0}=0$ and therefore $e_{2}=3$, so we have exactly 3 edges with both end-vertices colored (and hence no 4-cycle with all vertices colored). Note also, that since $n_{3}=2 t$, color 3 cannot be used on such edges.

We now check all different pairs of $u \sim v$ with $|f(u)|=|f(v)|=1$ to arrive at the contradiction. (Alternatively, we could denote $\mathcal{P}_{i}=\left\{u_{i}, v_{i}, u_{i+1}, v_{i+1}, u_{i-1}, v_{i-1}\right\}$ and $\gamma_{i}=\sum_{v \in \mathcal{P}_{i}}|f(v)|$ to see that $3 \leq \gamma_{i} \leq 4$ and $\sum_{i} \gamma_{i}=3 w(f)$ implies that $\gamma_{i}=4$ for exactly 3 consecutive $i$, and get the contradiction after some further inspection.) First, we check pairs $u_{i} \sim v_{i}$ with $i=1, \ldots, m-2 . \operatorname{Wlog} f\left(u_{1}\right)=f\left(v_{1}\right) \neq$ 0 . Then exactly one of $u_{0}, v_{0}$ is non-colored, say $f\left(u_{0}\right)=\emptyset, f\left(v_{0}\right) \neq \emptyset$. Also, exactly one of $u_{2}, v_{2}$ is non-colored:

- If $f\left(u_{2}\right)=\emptyset$, we have $f\left(v_{2}\right) \neq \emptyset$ and so $v_{0} v_{1}, v_{1} u_{1}, v_{2} v_{3}$ are the 3 edges with both end-vertices colored, therefore exactly one of $u_{i}, v_{i}$ is noncolored for all other $i$. This implies $f\left(v_{3}\right)=f\left(u_{4}\right)=$ $f\left(v_{5}\right)=\ldots=f\left(v_{n-2}\right)=f\left(u_{n-1}\right)=\emptyset$. Since $f(v)=\{3\}$ only for vertices with 3 non-colored neighbours, we have $\{3\}=f\left(u_{3}\right)=f\left(v_{6}\right)=\ldots=f\left(v_{m-1}\right)$. Now take wlog $f\left(v_{2}\right)=\{2\}$ and $f\left(u_{1}\right)=\{1\}$ and see this forces $\{2\}=f\left(v_{2}\right)=f\left(u_{5}\right)=\ldots=f\left(u_{1}\right)$, a contradiction. 


\begin{tabular}{|c|c|c|}
\hline$s$ & $\gamma_{r 3}(G)$ & $f\left(\begin{array}{llllllll}u_{0} & u_{1} & u_{2} & u_{3} & u_{4} & u_{5} & \cdots & u_{m-1} \\
v_{0} & v_{1} & v_{2} & v_{3} & v_{4} & v_{5} & \cdots & v_{m-1}\end{array}\right)$ \\
\hline 0 & $m+1$ & $\begin{array}{lllllllllllll}\emptyset & 2 & \emptyset & 1 & \emptyset & 3 & \ldots & \emptyset & 2 & \emptyset & 1 & \emptyset & 3 \\
1 & \emptyset & 3 & \emptyset & 2 & \emptyset & \ldots & 1 & \emptyset & 3 & \emptyset & 2 & 3 \\
\end{array}$ \\
\hline 1 & $m+2$ & $\begin{array}{llllllll}\emptyset & 2 & \emptyset & 1 & \emptyset & 3 & \ldots & 1 \\
1 & \emptyset & 3 & \emptyset & 2 & \emptyset & \ldots & 1 \\
\end{array}$ \\
\hline 2 & $m+1$ & $\begin{array}{lllllllll}\emptyset & 2 & \emptyset & 1 & \emptyset & 3 & \ldots & 0 & 2 \\
1 & \emptyset & 3 & \emptyset & 2 & \emptyset & \ldots & 1 & 3 \\
\end{array}$ \\
\hline 3 & $m$ & $\begin{array}{llllllllll}\emptyset & 2 & \emptyset & 1 & \emptyset & 3 & \ldots & 0 & 2 & 0 \\
1 & \emptyset & 3 & \emptyset & 2 & \emptyset & \ldots & 1 & 0 & 3 \\
\end{array}$ \\
\hline 4 & $m+1$ & $\begin{array}{lllllllllll}\emptyset & 2 & \emptyset & 1 & \emptyset & 3 & \ldots & 0 & 2 & 0 & 1 \\
1 & \emptyset & 3 & \emptyset & 2 & \emptyset & \ldots & 1 & 3 & 3 & 3 \\
\end{array}$ \\
\hline 5 & $m+1$ & $\begin{array}{llllllllllll}\emptyset & 2 & \emptyset & 1 & \emptyset & 3 & \ldots & 0 & 2 & 0 & 1 & 3 \\
1 & \emptyset & 3 & \emptyset & 2 & \emptyset & \ldots & 1 & 0 & 3 & 0 & 2 \\
\end{array}$ \\
\hline
\end{tabular}

Table 2: $\gamma_{r 3}(G)$-functions for $G=\operatorname{ML}(m)$, where $m=6 t+s \geq 2$.

\begin{tabular}{|c|c|c|c|c|}
\hline$s$ & $\gamma_{r 4}(G)$ & $f\left(\begin{array}{llllllll}u_{0} & u_{1} & u_{2} & u_{3} & u_{4} & u_{5} & \cdots & u_{m-1} \\
v_{0} & v_{1} & v_{2} & v_{3} & v_{4} & v_{5} & \cdots & v_{m-1}\end{array}\right)$ & $\gamma_{r 5}(G)$ & $f\left(\begin{array}{llllllll}u_{0} & u_{1} & u_{2} & u_{3} & u_{4} & u_{5} & \cdots & u_{m-1} \\
v_{0} & v_{1} & v_{2} & v_{3} & v_{4} & v_{5} & \cdots & v_{m-1}\end{array}\right)$ \\
\hline 0 & $\frac{4}{3} m+1$ & 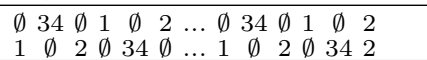 & $\frac{5}{3} m+1$ & 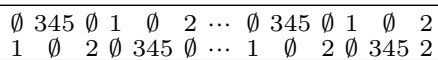 \\
\hline 1 & $\frac{4}{3} m+\frac{5}{3}$ & $\begin{array}{lllllllll}\emptyset & 34 & \emptyset & 1 & \emptyset & 2 & \ldots & 3 \\
1 & \emptyset & 2 & \emptyset & 34 & \emptyset & \ldots & 12 \\
\end{array}$ & $\frac{5}{3} m+\frac{4}{3}$ & $\begin{array}{lllllllll}\emptyset & 345 & \emptyset & 1 & \emptyset & 2 & \cdots & 3 \\
1 & \emptyset & 2 & \emptyset & 345 & \emptyset & \cdots & 12 \\
1 & & & & \end{array}$ \\
\hline 2 & $\frac{4}{3} m+\frac{4}{3}$ & $\begin{array}{lllllllll}\emptyset & 34 & \emptyset & 1 & \emptyset & 2 & \ldots & 0 & 34 \\
1 & \emptyset & 2 & \emptyset & 34 & \emptyset & \ldots & 1 & \\
1\end{array}$ & $\frac{5}{3} m+\frac{2}{3}$ & $\begin{array}{lllllllll}\emptyset & 345 & \emptyset & 1 & \emptyset & 2 & \cdots & 3 & 4 \\
1 & \emptyset & 2 & \emptyset & 345 & \emptyset & \cdots & 1 & 2 \\
\end{array}$ \\
\hline 3 & $\frac{4}{3} m$ & 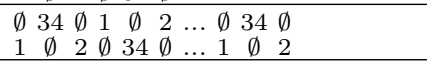 & $\frac{5}{3} m$ & $\begin{array}{cccccccccc}\emptyset & 345 & \emptyset & 1 & \emptyset & 2 & \cdots & \emptyset & 445 & \emptyset \\
1 & \emptyset & 2 & \emptyset & 345 & \emptyset & \cdots & 1 & \emptyset & 2 \\
\end{array}$ \\
\hline 4 & $\frac{4}{3} m+\frac{2}{3}$ & $\begin{array}{lllllllllll}\emptyset & 34 & \emptyset & 1 & \emptyset & 2 & \ldots & \emptyset & 34 & 0 & 1 \\
1 & \emptyset & 2 & \emptyset & 34 & \emptyset & \ldots & 1 & 0 & 2 & 2 \\
1\end{array}$ & $\frac{5}{3} m+\frac{1}{3}$ & 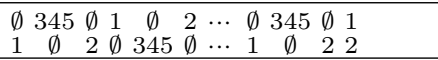 \\
\hline 5 & $\frac{4}{3} m+\frac{4}{3}$ & $\begin{array}{llllllllllll}\emptyset & 34 & \emptyset & 1 & \emptyset & 2 & \ldots & \emptyset & 34 & \emptyset & 1 & 4 \\
1 & \emptyset & 2 & \emptyset & 34 & \emptyset & \ldots & 1 & \emptyset & 2 & 3 & 2\end{array}$ & $\frac{5}{3} m+\frac{2}{3}$ & $\begin{array}{cccccccccccc}\emptyset & 345 & \emptyset & 1 & \emptyset & 2 & \cdots & \emptyset & 345 & \emptyset & 1 & 4 \\
1 & \emptyset & 2 & \emptyset & 345 & \emptyset & \cdots & 1 & \emptyset & 2 & 3 & 2\end{array}$ \\
\hline
\end{tabular}

Table 3: $\gamma_{r k}(G)$-functions of $G=\operatorname{ML}(m)$ for $k=4,5$ where $m=6 t+s \geq 2$.

- If $f\left(u_{2}\right) \neq \emptyset$, we have $f\left(v_{2}\right)=\emptyset$ and so $v_{0} v_{1}, v_{1} u_{1}, u_{1} u_{2}$ are the 3 edges with both end-vertices colored. Again exactly one of $u_{i}, v_{i}$ is noncolored for all other $i$ implying that $f\left(v_{m-1}\right)=\emptyset$, a contradiction, since $v_{m-1} \sim u_{0}$ and $f\left(u_{0}\right)=\emptyset$. so for all other $j$, we have exactly one of $u_{j}, v_{j}$ colored.

In similar fashion, we obtain a contradiction in all other cases. Therefore $\gamma_{r 3}(G)=m+2$ for $m \equiv 1$ $(\bmod 6)$.

For $k=4,5$, the proof is similar as the proof for prisms. For instance, we use Theorems 1.1 and 1.2 to obtain

$$
\left\lceil\frac{4}{3} m\right\rceil \leq \gamma_{r 4}(G) \leq\left\lceil\frac{4}{3} m\right\rceil+ \begin{cases}0, & m \equiv 3,4 \quad(\bmod 6) \\ 1, & m \equiv 0,2,5 \quad(\bmod 6) \\ 2, & m \equiv 1 \quad(\bmod 6)\end{cases}
$$

Then we find an appropriate 4-RDF (see Table 3) and apply different combinatorial arguments to prove that lower weight is not possible. We omit further details.

Corollary 4.6. The only connected 3-rainbow domination regular Cayley graphs over abelian groups are prisms $\operatorname{Pr}(m)$, where $m \equiv 0(\bmod 6)$, and Möbius ladders $\operatorname{ML}(m)$, where $m \equiv 3(\bmod 6)$.

\section{Concluding remarks}

The investigations of $k$-rainbow domination numbers for $d$-regular graphs from previous sections can be naturally generalized to some other classes of cubic graphs or to specific classes of $d$-regular graphs with $d \geq 4$. However, determining exact $k$-rainbow domination numbers for all 4-regular Cayley graphs over abelian groups might turn out to be quite difficult already.

It would also be interesting to obtain further classification of $d$-RDR graphs. For two more examples, see Figure 5. It is easy to check that the tesseract graph $Q_{4}$ is a 4-RDR graph, and the wreath graph $G=C_{m}\left[2 K_{1}\right]$ (the lexicographic product of a cycle with 2 isolated vertices) is a 4-RDR graph for all $m \geq 3$ with $m \equiv 0(\bmod 4)$.

We observe that in both cases, the graphs are Cayley graphs over abelian groups, namely $Q_{4} \cong$ $\operatorname{Cay}\left(\mathbb{Z}_{4},\left\{e_{1}, e_{2}, e_{3}, e_{4}\right\}\right)$ and $C_{m}\left[2 K_{1}\right] \cong \operatorname{Cay}\left(\mathbb{Z}_{m} \times \mathbb{Z}_{2},\{( \pm 1,0),( \pm 1,1)\}\right)$. We can thus ask the following questions: 

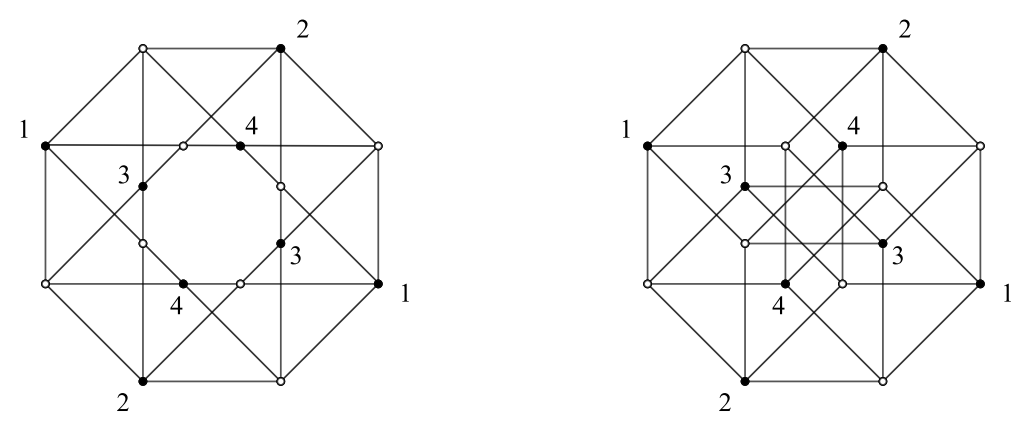

Figure 2: Two 4-RDR graphs, the wreath graph $C_{4}\left[2 K_{1}\right]$ and the tesseract $Q_{4}$, with indicated values of their $\gamma_{r 4}$-functions.

- Question 1: Are there any $d$-RDR graphs that are not obtained as Cayley graphs over some abelian group?

- Question 2: More generaly, are there any $d$-RDR graphs that are not vertex transitive?

\section{Acknowledgments}

This work is supported in part by the Slovenian Research Agency (ARRS), research program P1-0285 and research projects J1-9108, J1-1694, J1-1695. The author also thanks to Primož Šparl for suggesting the problem.

\section{References}

[1] N. Bray, E. W. Weisstein, Domination Number, from MathWorld-A Wolfram Web Resource. http://mathworld.wolfram.com/DominationNumber.html

[2] B. Brešar, M.A. Henning, D.F. Rall, Rainbow domination in graphs, Taiwanese J. Math. 12 (2008), 213-225.

[3] B. Brešar, T. Kraner Šumenjak, On 2-rainbow domination in graphs, Discrete Applied Mathematics 155 (2007), $2394-2400$.

[4] G. J. Chang, J. Wu, X. Zhu, Rainbow domination on trees, Discrete Applied Mathematics, Volume $158(2010), 8-12$.

[5] S. Fujita, M. Furuya, C. Magnant, General Bounds on Rainbow Domination Numbers, Graphs and Combinatorics 31 (2015) $601-613$.

[6] M. Furuya, M Koyanagi, M. Yokota, Upper bound on 3-rainbow domination in graphs with minimum degree 2, Discrete Optimization 29 (2018), 45-76.

[7] B. Hartnell, D.F. Rall, On dominating the Cartesian product of a graph and $K_{2}$, Discuss. Math. Graph Theory 24 (2004) 389-402.

[8] T. Kraner Šumenjak, D. F. Rall, A. Tepeh, Rainbow domination in the lexicographic product of graphs, Discrete Applied Mathematics 161 (2013), 2133 - 2141.

[9] Z. Shao, M. Liang, C. Yin, X.Xu, P. Pavlič, J. Žerovnik, On rainbow domination numbers of graphs, Information Sciences 254 (2014), 225 - 234.

[10] Z. Shao, H. Jiang, P. Wu, S. Wang, J. Žerovnik, X. Zhang, J.B. Liu, On 2-rainbow domination of generalized Petersen graphs, Discrete Applied Mathematics 257 (2019), 370 - 384. 
[11] Z. Stepien, A. Szymaszkiewicz, L. Szymaszkiewicz, M. Zwierzchowski, 2-Rainbow domination number of $C_{n} \square C_{5}$, Discrete Appl. Math., 170 (2014), 113 - 116.

[12] Wang Y., Wu K., A tight upper bound for 2-rainbow domination in generalized Petersen graphs, Discrete Appl. Math., 161 (2013), 2178 - 2188. 